\title{
Experiments on Normative Population Projek- tion: Optimizing the Long-term Activity Rate
}

By HANNU KULOKARI

Computing Center, University of Helsinki

This study is a result of spare-time activity at the Computing Center, University of Helsinki, whose facilities have made the work possible. For the writing of this article the author has been awarded a grant by the Finnish Academy, which is duly appreciated.

\section{Introduction: The methodology of normative population projections}

The usual population projections strive to be probable or surprise-free, or to give an impression about what would happen in the population, if certain assumptions concerning the behaviour of the components came true. It is eustomary to examine how population development would be affected by various combinations of the component estimates.

A projection becomes normative when one specifies what should happen in the population, and the problem is then to determine how this end could be achieved.

In recent years normative projections have become quite common in demography. The central point of interest has been zero or stable population growth: how it could be achieved. The independent variable, in terms of which the alternatives are stated, has usually been fertility. The best known examples of this kind of study are those by Bourgois-Pichat \& Taleb (1970), Frejka (1968, 1973) and, in the Nordic countries, Matthiessen (1974). These calculations have shown that an immediate halt in population growth would mean exaggerated and improbable oscillations in fertility level and age structure, and that a slower and more even fertility decline would allow the population to continue to grow for a considerable time.

In all these calculations, the analyst himself constructs all the alternatives, which are then tested to determine whether they lead to the desired or acceptable end result. This is not satisfactory methodologically, because it usually leads to serious omissions. For instance, we have not often seen projections which would allow fertility to rise after it has fallen. One might think this would be impossible according to the demographic theory, what little of it exists. 
An indication of the reality of this so far latent omission problem is a recent Review Symposium in Demography (May 1974): Reviewing Frejka's book 'The Future of Population Growth', the reviewers (Masnick, Bourgois-Pichat, Schutz) listed and actually even calculated several new alternatives not examined in Frejka's analysis.

More rigour for this kind of analysis, 'how the good could be achieved', is obviously needed. In many other fields of research a practice has long since been established, where one first defines 'good' in operational terms and then examines all the logically possible and reasonable alternatives in order to find the 'best'. If the problem structure is suitable, one can utilize the efficient methods of mathematical optimization, and thus avoid actually enumerating all possibilities, which would often prove overtaxing. This way the analyst is relieved of the routine task of generating alternatives, and may concentrate on the analysis.

This is what in an experimental way is being attempted in the present article. The 'good' to be achieved is not, however, zero population growth, but an optimal level of activity in the population, which is politically a more pressing problem in a country like Finland.

\section{The problem}

Let us consider a fixed-length future of a closed population. The development of age-specific mortality is assumed known, as are the upper and lower limits for age-specific fertility in each intermediate period. Furthermore it is specified just how much at most fertility may change between two successive intermediate periods.

It is desired to keep the activity rate, defined as the proportion of the population of working age, as high as possible in the future, either on the average or period by period.

How should fertility develop within its specified limits?

Is the activity rate a relevant measure for labour supply? Is not the demand usually expressed in absolute terms? Economists in particular seem to attach more importance to the absolute size of the labour force.

But, in a long-term analysis, the economist's way of treating the national product as an autonomous variable is not appropriate. It would be putting the cart before the horse to state that, because the national product obviously must grow at this particular rate and productivity improve in a certain way, then the labour force must be of that particular size. Yet this statement is often made, especially in the mass media. Much of the current talk concerning the future shortage of labour is based on the tacit assumption that production must continue to increase forever. Why, if the population does not grow? (Of course, Finland does have foreign debts which must be settled sometime.) 


\section{Criteria for solution}

Some suggested criteria for the 'goodness' of an alternative population development path:

MAXIMEAN: The average activity rate weighted by total population.

MAXIMIN: The lowest yearly activity rate of an alternative. Maximizing this means keeping the activity rate continually as high as possible.

MINIDIF: The difference between highest and lowest yearly activity rate. Minimizing this criterion means keeping the activity rate as constant as possible.

Because the best distinctive characteristic of 'good' is the way it differs from 'bad', we define for these criteria also their opposites, MINIMEAN, MINIMIN and MAXIDIF. Each of these represents one type of 'bad' population development.

\section{The method of solution}

The aim here is to redefine the problem situation in a form which clearly brings out the essential characteristics of a potential solution. The actual solution algorithm is not described here. Full details as well as the FORTRAN program used in computation will be made available upon request by the author.

Define first the highest and lowest values the total population may take at each particular time in the future. Call these $\mathrm{MAX}_{t}$ and $\mathrm{MIN}_{\mathrm{t}}$. These values can be found by conventional projections using the highest and lowest fertility alternatives.

Divide each range $\left(\mathrm{MAX}_{\mathrm{t}}-\mathrm{MIN}_{\mathrm{t}}\right)$ into a suitable number of intervals of equal length, the end points of which form the values $\mathrm{P}_{t, \mathrm{i}}$, that the total population may possibly take at each future time. The inaccuracy resulting from discretization depends on the number of intervals and the size of the range. In the experimental calculations 49 intervals were used and the maximum range was 6 millions. The average error due to discretization is thus about 30000 . Because of this numerical inaccuracy, the results are not presented in tabular from in this article.

The required computational effort depends quadratically on the number of intervals. So one must define in advance how much accuracy is really needed.

Now we have a set of population sequences. Figure 1 describes in a schematical the structure of the solution space composed of these sequences. The heavier line represents one possible sequence. Our criterion assigns a value for each of these sequences. The task is to select the sequence having the optimal value. Please note that if the number of intervals is $\mathbf{C}$ and the length of the projection period is $\mathrm{N}$, there are $(\mathrm{C}+1)^{\mathrm{N}}$ possible sequences. With $\mathrm{C}=$ 49 and $\mathrm{N}=20$, this gives approximately $10^{34}$ different sequences, which is too much for even a computer to enumerate. 
Figure 1. Structure of the solution space.

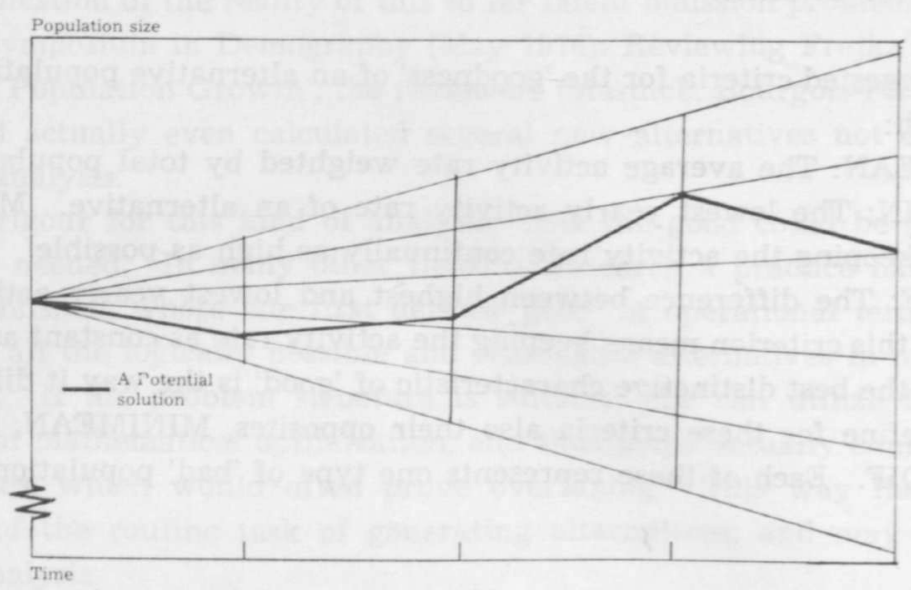

A simplification is possible by noting that not all the sequences are feasible, requiring either too high or too low fertility at some intermediate periods. This does not, however, sufficiently reduce the size of the problem.

Yet by dynamic programming we are able to examine all the sequences without actually enumerating them. The theory of dynamic programming is presented in most textbooks on mathematical programming and operations research. Special texts, such as Jacobs (1967) also abound.

The principle of DP is to reduce problem size by stagewise suboptimizing. The method is, however, different from the following trivial 'stagewise processing', which does not work: It could occur to someone to start from the beginning and proceed stage by stage to that succeeding population optimizing the value of the criterion at that stage. For example, being at stage $t$ with population $\mathrm{K}_{\mathrm{t}}$ (size and structure known), one would select that total population size $\mathrm{P}_{\mathrm{t}+1, \mathrm{i}}$ which, when achieved by a suitable number of births, would have the highest activity rate. This method of solution does not guarantee optimal solution, because at each stage all those sequences not passing through the selected total population size are excluded from further consideration. So only a few different complete sequences are evaluated and compared, and they differ only in their last stages.

\section{An experiment}

\subsection{The problem operationalized}

The population of Finland was studied for a time period of 100 years 1970 2070. Without sacrificing anything essential, the problem was simplified in the following two ways:

- Age-specific mortality stays constant at the level recorded in 1966-70. 
- The relative sizes of the age-specific fertility rates stay constant as in 1966 70 , so that the values corresponding to different net reproduction rates can be calculated using a constant multiplier.

The high and low limits for fertility were defined in terms of the (period) net reproduction rate (NRR). The NRR should stay between 0.8 and 1.2. Furthermore it was required, that the NRR shall not change more than $20 \%$ in a five year period.

The activity rate was defined as the percentage proportion of people aged 20 to 59 years.

All six criteria (three 'good' and three 'bad') were optimized. In addition two conventional projections were produced, corresponding to the high and low limits of fertility, named HIGH and LOW respectively.

\subsection{The data}

The initial population used was the Finnish census population 31.12.1970 by five-year age groups. Death risks correspond to the Finnish life table covering the period 1966-70. The age-specific fertility rates date also to 1966-70. The source of all these data was the Statistical Yearbook of Finland, 1973 edition.

The sexes were combined, instead of adopting the more common practice of analyzing the female population only. (The sexes could have been kept separate, of course, but this would have duplicated the computational effort without adding anything essential in return.) The fertility rates were adjusted according to the assumption that the sex ratio within the fertile age groups corresponds to that of the stationary population.

Table 1 contains some summary statistics describing the initial situation. It is worth noting that, compared to the stationary population, the initial population is rather young and inactive. So the future will probably have something better to offer.

$\mathrm{Table}$ 1. Summary statistics of the basic data.

\begin{tabular}{|c|c|}
\hline Total population 1970 & 4598336 \\
\hline Net reproduction rate & .989 \\
\hline Female NRR & 1.004 \\
\hline $\mathrm{e}_{0}$ & 69.8 \\
\hline$e_{10}$ & 61.2 \\
\hline $\mathrm{e}_{50}$ & 24.1 \\
\hline \multicolumn{2}{|l|}{ Age structure } \\
\hline Actual & Stationary $\%$ \\
\hline $0-19$ & 28.1 \\
\hline $20-59$ & 53.1 \\
\hline $60-$ & 18.8 \\
\hline
\end{tabular}




\subsection{Preliminary observations}

A conventional way to study the future age structure of a population is to use the stable population theory. Table 2 gives the activity rates in stable populations corresponding to some thinkable values of the NRR.

The stable populations suggest that the maximum activity rate would be attained at NRR values below the replacement level, the best tabulated value being $53.6 \%$ at $\mathrm{NRR}=0.8$. The stable condition is, however, still to be achieved.

$\mathrm{T}$ a ble 2. Activity rates (AR) in stable populations corresponding to selected levels of net reproduction rate (NRR).

$\begin{array}{cccc}\text { NRR } & \text { AR } & \text { NRR } & \text { AR } \\ 0.5 & 50.3 & 0.9 & 53.5 \\ 0.6 & 52.2 & 1.0 & 53.1 \\ 0.7 & 53.2 & 1.1 & 52.6 \\ 0.8 & 53.6 & 1.2 & 51.8\end{array}$

Figure 2. Development of the size of the population.

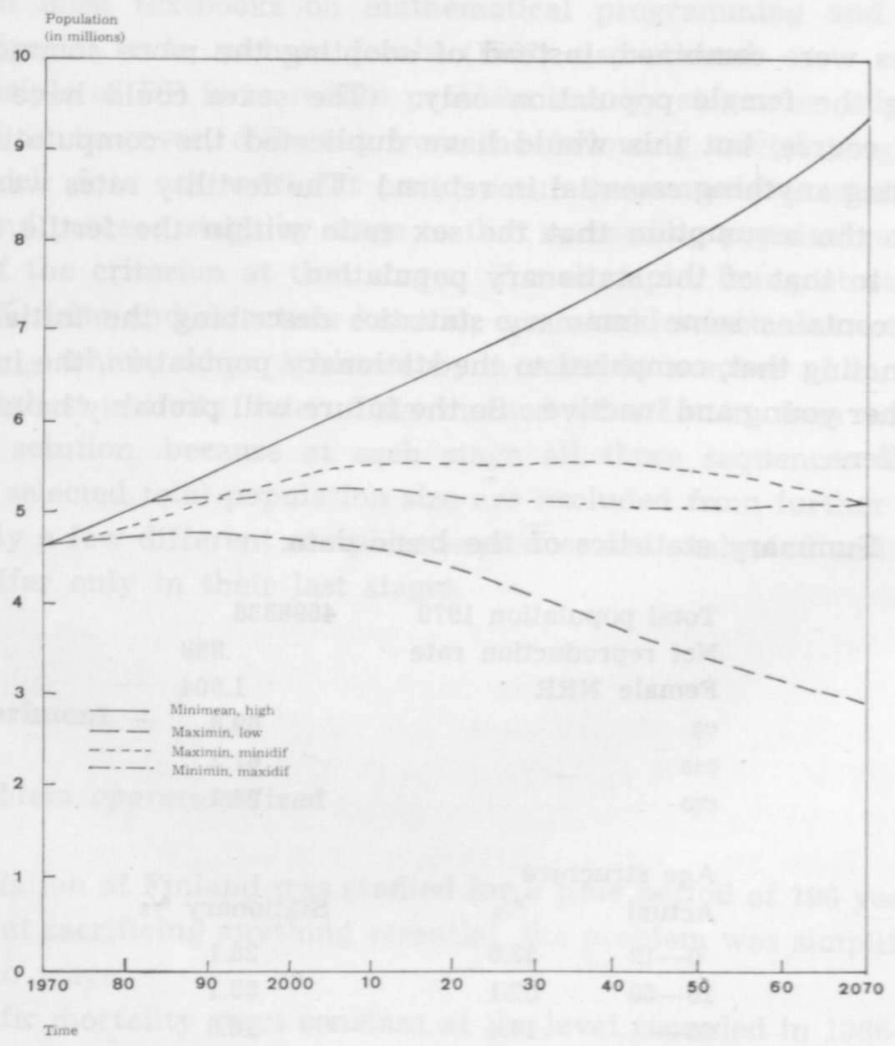




\subsection{The results: Initial comments}

The development of the total population, activity rate and NRR according to the different alternatives is described pictorially in figures 2,3 and 4 , respectively.

Figure 3. Development of the percentage proportion of age groups 20 to 59 (activity rate).

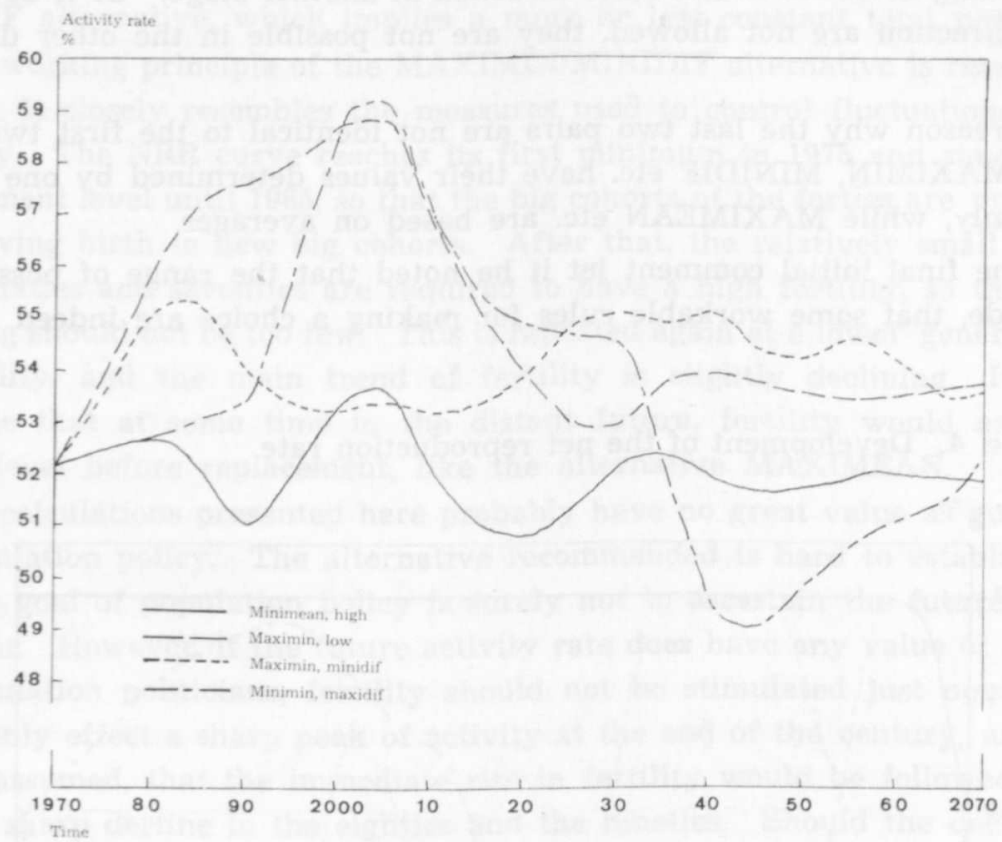

The most eye-catching detail in these graphs is that the following pairs of alternatives are identical:

- MAXIMEAN and LOW

- MINIMEAN and HIGH

- MAXIMIN and MINIDIF

- MINIMIN and MAXIDIF

So we have only four distinct alternatives. This deserves comment. The first two pairs are identical, because:

- As was noted in the preliminary observations, the 'best' and 'worst' stable populations within the range of alternatives correspond to NRR:s of 0.8 and 1.2 , that is, to the alternatives LOW and HIGH

- The period of 100 years is apparently so long, that the stable condition can be achieved sufficiently early within this period so that the 'stable' years 
outweigh the initial 'unstable' years (or at least weigh enough to determine the solution). This was, it must be confessed, somewhat surprising to the author. Perhaps a shorter period would have allowed the MAXIMEAN and MINIMEAN criteria to stand out as independent alternatives.

The identity of the two last alternative pairs, MAXIMIN/MINIDIF, MINIMIN/MAXIDIF demonstrates the inherent stability of the population system: A deviation in one direction at one stage of the population process necessitates an offsetting deviation in the other direction at another stage. Or if deviations in one direction are not allowed, they are not possible in the other direction either.

The reason why the last two pairs are not identical to the first two pairs, is that MAXIMIN, MINIDIF etc. have their values determined by one or two points only, while MAXIMEAN etc. are based on averages.

As the final initial comment let it be noted that the range of possibilities is so wide, that some workable rules for making a choice are indeed needed.

Figure 4. Development of the net reproduction rate.

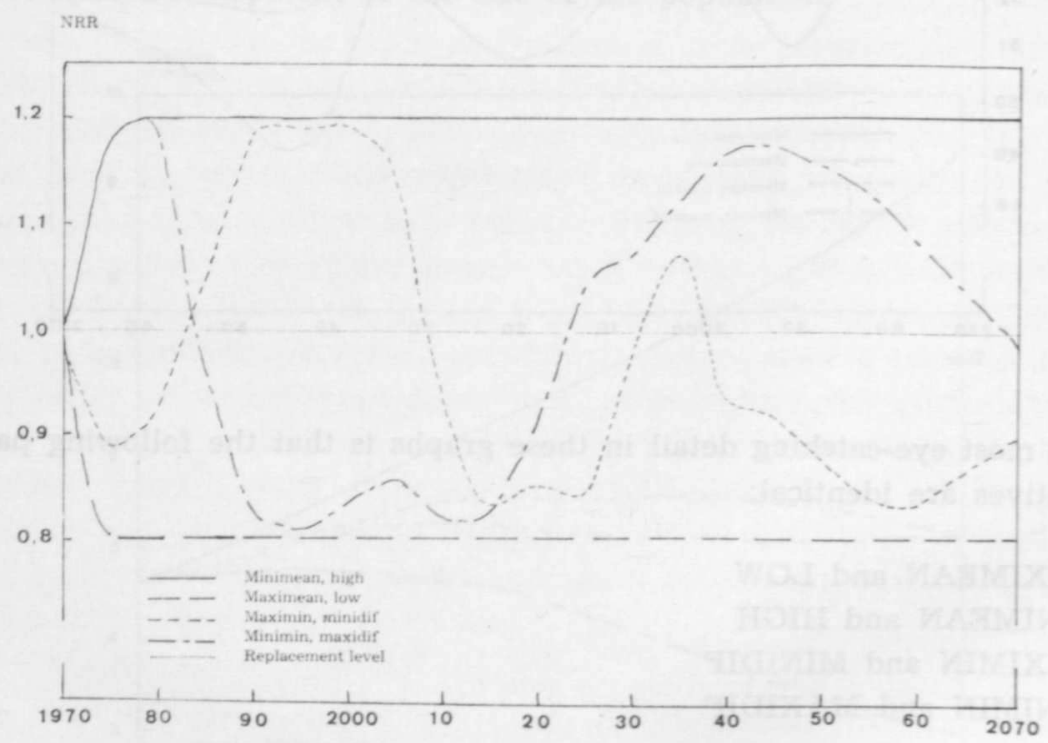

\subsection{The results: Discussion}

Although the calculations were purely experimental in nature, some trends are so clear that they cannot avoid comment.

The amplitude of the activity rate in the alternative pair MAXIDIF/ MINIMIN is intolerably large, a total of 10 percentage points. This would surely 
bring about adjustment difficulties for society. So there lie some dangers in the future, things to be avoided.

Measured by the criterion MAXIMIN, the actual MAXIMIN-alternative is very little better than the MAXIMEAN (or LOW) alternative. Furthermore, even on this criterion, the MAXIMEAN alternative is better until the year 2020 . However, the MAXIMEAN alternative means a continual decrease in the population, which may prove unpleasant and have disastrous effects on the economy by reducing the home market and thus removing some of the economies of scale. So, at least the author is more in favour of the MAXIMIN/ MINIDIF alternative, which implies a more or less constant total population.

The working principle of the MAXIMIN/MINIDIF alternative is remarkably simple. It closely resembles the measures used to control fluctuations in the economy. The NRR curve reaches its first minimum in 1975 and stays below replacement level until 1985, so that the big cohorts of the forties are prevented from giving birth to new big cohorts. After that, the relatively small cohorts of the sixties and seventies are required to have a high fertility, so that their offspring should not be too few. This is repeated again at a lower general level of fertility, and the main trend of fertility is slightly declining. It seems plausible that at some time in the distant future, fertility would assume a steady level before replacement, like the alternative MAXIMEAN.

The calculations presented here probably have no great value as guidelines for population policy. The alternative recommended is hard to establish, and the sole goal of population policy is surely not to ascertain the future supply of labour. However, if the future activity rate does have any value of interest for population politicians, fertility should not be stimulated just now. That would only effect a sharp peak of activity at the end of the century, and only if it is assumed, that the immediate rise in fertility would be followed by an equally sharp decline in the eighties and the nineties. Should the decline not occur (as in alternative MINIMEAN/HIGH), the activity rate would never rise significantly above the current level and most of the time would stay at a much lower level. And should the decline occur or not, the next century would be very inactive.

\section{Conclusion}

Considering a closed population with a known range of fertility development alternatives, the problem was defined as to how fertility should develop in order to keep the activity rate of the population as good as possible. After defining some criteria for 'good' and 'bad' and outlining a method of solution, some experimental calculations were made on the Finnish data for the period 1970 - 2070. Some justification for tentative recommendations was found. The recommended alternative would quarantee a minimum activity level of $53 \%$ of the population in age groups 20 through 59 throughout the period and would 
require the following fertility development: Low in the seventies, high until 2005, low until 2030, peak at 2035 and low afterwards. This recommendation partially contradicts current thinking about population policy.

\section{References}

Bourgois-Pichat \& Taleb: Un taux d'accroissement nul pour les pays en voie de développement en 2000: Rêve ou réalité? Population, Vol. 25, 1970.

Frejka: Reflections on the Demographic Conditions needed to Establish a U.S. Stationary Population Growth. Population Studies, Vol. 22, 1968.

Frejka: The Future of Population Growth: Alternative Paths to Equilibrium. New York 1973.

Jacobs: An Introduction to Dynamic Programming. London 1967

Matthiessen: Population Stabilization in the Nordic Countries. Scandinavian Population Studies, Vol. 3, 1974.

Review Symposium (On Frejka (1973)) Demography, Vol. 11, nr 2, 1974. 